\title{
Efficacy of breast shielding during head computed tomography examination
}

\author{
Nika Zalokar, Nejc Mekis \\ University of Ljubljana, Faculty of Health Sciences, Medical Imaging and Radiotherapy Department, Ljubljana, Slovenia \\ Radiol Oncol 2021; 55(1): 116-120. \\ Received 20 May 2020 \\ Accepted 16 June 2020 \\ Correspondence to: Nejc Mekiš, Ph.D., Zdravstvena pot 5, SI-1000 Ljubljana, Slovenia. E-mail: nejc.mekis@zf.uni-lj.si \\ Disclosure: No potential conflicts of interest were disclosed.
}

Background. Female breasts are exposed to scattered radiation regardless of not being included in the primary field during head CT. This study aimed to investigate whether the use of lead shielding is beneficial in dose reduction to the breasts during head CT.

Patients and methods. The study was performed in two different hospitals on two different $\mathrm{CT}$ units and included 120 patients. Half of the measurements $(n=60)$ was conducted without the use of lead shielding and the other half $(n=60)$ with the use of lead shielding of $0.5 \mathrm{~mm}$ equivalent thickness.

Results. Significant skin dose reduction to the breasts during head $\mathrm{CT}$ in both hospitals with the use of lead shielding was discovered; $81 \%$ (338.2 $\pm 43.7 \mu \mathrm{G} y$ to $64.3 \pm 18.8 \mu \mathrm{Gy}$ ) in Hospital A and 74\% (from 253.1 $\pm 35.1 \mu \mathrm{Gy}$ to $65.3 \pm$ $16.9 \mu \mathrm{Gy})$ in Hospital B.

Conclusions. Considering the assumed carcinogenic effect of low doses of radiation, high frequency of the head CT scans and the significant reduction of radiation doses to the highly radiosensitive breasts, the use of lead shielding is highly recommendable.

Key words: scatter radiation; head CT; lead shielding; breasts; dose reduction

\section{Introduction}

Computed tomography (CT) is a valuable diagnostic tool for many conditions. ${ }^{1}$ According to the International Atomic Energy Agency (IAEA) an individual receives $2.4 \mathrm{mSv}$ of the total annual dose of natural radiation ${ }^{2}$ and the typical effective radiation dose received during a standard CT scan ranges from 1 to $14 \mathrm{mSv}{ }^{3}$ Head CT is one of the most frequently performed examinations ${ }^{4,5}$ and accounts for $50 \%$ of all CT scans and $25 \%$ of the collective radiation dose from CT. ${ }^{5}$ High effective doses received during $\mathrm{CT}$ examinations raise concern $\mathrm{s}^{1,6}$, so an effort to minimize it is crucial. ${ }^{1}$ Due to concerns over radiation exposure, the very valuable principle "as low as reasonably achievable" (ALARA) was established to stress the importance of minimizing radiation exposure. ${ }^{7}$ Many $\mathrm{CT}$ shielding techniques have been developed as a result, among them radiation protection shields, which are one of the simplest mechanisms by which radiation dose can be reduced. ${ }^{3}$

The use of lead shielding is a long-accepted method to minimize the dose from scatter radiation in radiographic procedures..$^{1,8}$ In head $\mathrm{CT}$, the head as the main target under investigation is included in the primary beam, while radiosensitive organs such as the breasts are located outside the primary beam. Radiosensitive structures that lie in the primary beam cannot be easily protected, while the organs outside the primary beam, which are mainly exposed to scattered radiation, can be easily protected against it. Scattered radiation distributes caudally through patient's neck thereby inevitably exposing the breasts. However, scattered radiation produced in gantry and patient's head reaching the breasts from outside can be effectively diminished by the lead shielding. ${ }^{8}$ Radiation dose 
to the breasts is significant enough to be a matter of concern ${ }^{9}$ regardless of all the current dose reduction techniques. ${ }^{5}$

Female breast tissue is highly sensitive to radiation's carcinogenic effects. Its tissue weighting factor has risen from 0.05 to $0.12 .{ }^{7}$ The exposure of breasts to ionising radiation is a strong risk factor for breast cancer. The study by Preston et al. ${ }^{10}$ showed that the increased risk is directly proportional to the radiation dose received and inversely related to age at irradiation. The use of radiation shields, if applied properly, is one of the simplest implementations by which radiation dose can be diminished without the negative impact on diagnostic image quality. ${ }^{3}$

The benefit of shielding to radiosensitive organs in many diagnostic procedures has been acknowledged in many studies..$^{1,5,8,11-13}$ Brnić et al. ${ }^{8}$ recommend lead shielding of the breasts during head CT since a recognizable reduction (57\%) of the exposure was found. The study by Williams and Adams $^{5}$ also discovered a significant dose reduction (58-47\%) with a lead bib protecting the thyroid. Beaconsfield et al. ${ }^{11}$ also claim a significant dose reduction (76\%) during head CT due to lead shielding of breasts. However, Brnić et al. ${ }^{8}$ measured the exposure with thermoluminescent dosimeters (TLD) during shielding of only one breast while the other remained unshielded, the study by Williams and Adams ${ }^{5}$ performed measurements while investigating the benefit of thyroid shielding on an anthropomorphic phantom and Beaconsfield et al. ${ }^{11}$ have performed only 20 measurements with the use of breast shielding during axial head CT examination.

For the above-mentioned reasons and given the high frequency of head CT scans and high sensitivity of breasts to ionising radiation, the purpose of this study was to determine the effectiveness of breast shielding during the routine helical head CT scan in real clinical practice with an electronic dosimeter and a lead apron of $0.5 \mathrm{~mm}$ lead equivalent, which is the most commonly used protective shield in Slovenia. Lead shielding is not routinely used in the breast area during head CT examination so the results could clarify the dilemma whether the shielding is beneficial in protecting the breasts. Possible changes in clinical practice might be expected in the future.

\section{Patients and methods}

The study was performed in two major hospitals in Slovenia. The sample size was calculated based on the study performed by Brnić et al. ${ }^{8}$, with the G*Power 3.1 (Allgemeine Psychologie und Arbeitspsychologie, University Dusseldorf) programme. 120 female patients referred to a spiral head CT scan participated in our study (age range 23-92 years with average age of $67.9 \pm 16.7$ years). 60 measurements were conducted in Hospital A on Siemens Somatom Definition (Siemens Medical Systems, Germany) CT unit and 60 measurements in Hospital B with Toshiba Aquilion 64 slice (Toshiba Medical Systems, Otawara, Japan) CT unit. All results of the quality assurance tests for both CT units, performed prior to the study by the medical physicist, were under the remedial level. Half of the measurements in each hospital $(n=30)$ were performed with the use of lead shielding and half of the measurements $(n=30)$ without the lead shielding. Randomized sampling was used in this study.

The Unfors EDD 30 (Unfors, Sweden) unit was used to measure the skin dose at the surface of the breast in real time during CT of the head. According to the manufacturer, the dosimeter offers a dose range from $1 \mathrm{nGy}$ to 9999 Gy with the start trigger level at $0.05 \mathrm{mSv} / \mathrm{h}$. The manufacturer also claims that the EDD 30 's inaccuracy is $\pm 6 \%$ at calibration point with $80 \mathrm{kVp}, 2 \mathrm{~mm}$ of $\mathrm{Cu}$ and 4 $\mathrm{mm}$ of Al. The dosimeter was tested and showed the best and consistent response of the dosimeter when it was in alignment to the horizontal plane and the cable of the dosimeter was positioned away from the source of scattered radiation (not contained in the scanning area). The dose was read on the display.

Acquisition parameters are shown in Table 1. Same protocols as used in clinical practice in each hospital were used during the study.

The sensor of the dosimeter was attached to the centre of the breast in the alternating order, in a way that the dosimeter was always in alignment with the examination table to ensure minimal effect of the angulation dependence of the dosimeter. The breast area was wrapped with lead apron (Mavig, Germany) of $0.5 \mathrm{~mm}$ equivalent lead

TABLE 1. Acquisition parameters

\begin{tabular}{lcc}
\hline Hospital & A & B \\
\hline Pitch & 0.55 & 0.656 \\
Rotation time (s) & 1 & 0.6 \\
kV & 120 & 120 \\
mA & 480 & 200 \\
\hline
\end{tabular}


thickness as tightly as possible around the patient (shoulders were not draped). The example of the shielding position is presented on Figure 1 using an anthropomorphic phantom.

Our study was approved by the hospital's Ethics Committee and by the National Medical Ethics Committee prior to the initiation. A written informed consent was obtained from all patients. All the measurements were processed with the IBM SPSS STATISTICS version 25 (IBM, USA). The Shapiro-Wilk test was used to check the normal distribution of the sample. A student T-test was performed to determine the statistical significance of the difference of the skin dose between the shielded and unshielded breasts in each hospital. A significance of $p<0.05$ was used for all the tests.

\section{Results}

In Hospital $\mathrm{A}$ the mean skin dose to the breast was $338.2 \pm 43.7 \mu \mathrm{Gy}$ without the lead shielding and $64.3 \pm 18.8 \mu \mathrm{Gy}$ when the lead shielding was used. The difference between the doses was statistically significant $(\mathrm{p}<0.001)$ with the dose reduction of approximately $81 \%$. In Hospital B, the mean skin dose to the unprotected breasts was $253.1 \pm$ $35.1 \mu \mathrm{Gy}$ compared to $65.3 \pm 16.9 \mu \mathrm{Gy}$ to the breasts protected with the lead shielding. The dose reduction of $74 \%$ was statistically different $(p<0.001)$. Table 2 and Figure 2 summarize the results.

\section{Discussion}

This study provides evidence, that lead shielding significantly reduced the amount of scatter radiation to the breasts in both Hospitals.

Radiosensitive structures that lie in the primary field cannot be easily protected, meanwhile the structures outside the CT scanning plane, such as the breasts during the head CT examination, can be easily protected against the scatter radiation. During the head CT examination the use of lead shielding can significantly reduce the exposure

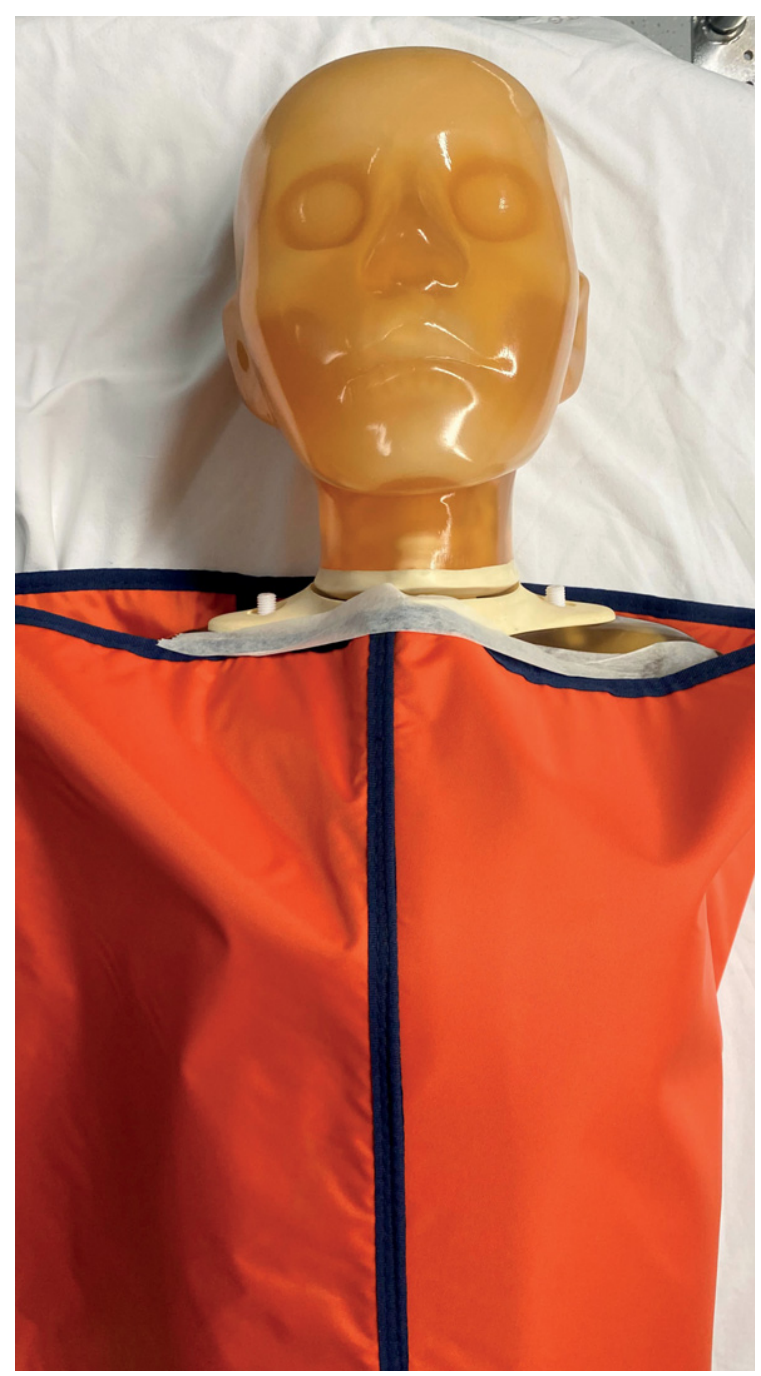

FIGURE 1. Presentation of the shielding position used in the study.

from scattered radiation generated in patient's head and in gantry. 8,14

Our results showed that the breast exposure in Hospital $\mathrm{A}$ and $\mathrm{B}$ was reduced by $81 \%$ and $74 \%$, respectively, with the use of $0.5 \mathrm{~mm}$ lead shielding. Nevertheless, a noticeably higher mean skin dose to breasts without the shielding in Hospital A, compared to Hospital B has been detected. This

TABLE 2. Mean measured skin dose to the breasts in both investigated hospitals

\begin{tabular}{lccc} 
& \multicolumn{2}{c}{ Hospital A } & \multicolumn{2}{c}{ Hospital B } \\
\cline { 2 - 4 } & unshielded & shielded & unshielded \\
\hline Mean skin dose to breast $(\mu \mathrm{Gy})$ & $338.2 \pm 43.7$ & $64.3 \pm 18.8$ & $253.1 \pm 35.1$ \\
Dose reduction & & $81 \%$ & $65.3 \pm 16.9$ \\
\hline
\end{tabular}


difference might be the result of the higher $\mathrm{mA}$ setting, longer scan lengths (were not noted) and the angular dependence of the dosimeter. Further investigation could be made.

The study from Beaconsfield et al. ${ }^{11}$ reported an average $76 \%$ dose reduction using a $0.5 \mathrm{~mm}$ lead equivalent thickness, which is similar to our study. They used the axial imaging technique of the head CT examination and not the spiral, as did we in our study. The axial imaging technique was also used in the study by Brnić et al. ${ }^{8}$, which reported lower reduction in dose; $57 \%$. However, their study used a $0.35 \mathrm{~mm}$ lead shield, and only one breast was shielded during the measurements, so a lower dose reduction is expected. However, Williams and Adams $^{5}$ did use the same CT imaging technique - the spiral scanning. They have investigated the impact of thyroid shielding on dose to the patient during head CT. They have measured a 58\% dose reduction on the thyroid during the spiral head CT examination. The thyroid, however, cannot be protected from the $\mathrm{X}$-rays scattered from within the patient. ${ }^{11}$ All mentioned studies used thermo-luminescent dosimeters to measure the radiation dose. In contrary, our study used an electronic dosimeter so the skin dose on the breasts was read out on the display as soon as the scanning was finished.

The International Commission on Radiological Protection (ICRP) has published new tissue weighting factors for the breast and other organs in 2007. The breast tissue weighting factor was increased from 0.05 to $0.12 .{ }^{7}$ One of the strongest risk factors for breast cancer is the radiation exposure at a young age. ${ }^{10,15}$ According to the ALARA principle all effort should be made to reduce irradiation of the breasts using shielding. In addition to breast shielding some other radiosensitive tissues will also be protected against the external scatter radiation, particularly bone marrow $(42 \%$ of bone marrow is found in the thorax). ${ }^{8,11}$ Based on the ICRP1037, bone marrow is a radiosensitive organ with the same tissue weighting factor as the breasts -0.12 , however, the effect of shielding on the bone marrow was not investigated in our study.

The study was conducted on female patients and not on an anthropomorphic phantom as the study by Williams and Adams ${ }^{5}$, so the measurements were performed on real body tissue in real clinical circumstances. In addition, patient positioning and diverse body geometry of the patients influenced the shielding possibilities. Shielding in the present study was wrapped around the chest of the patient so both breasts were shielded simultaneously and not one breast only like in the study by Brnić et al. ${ }^{8}$

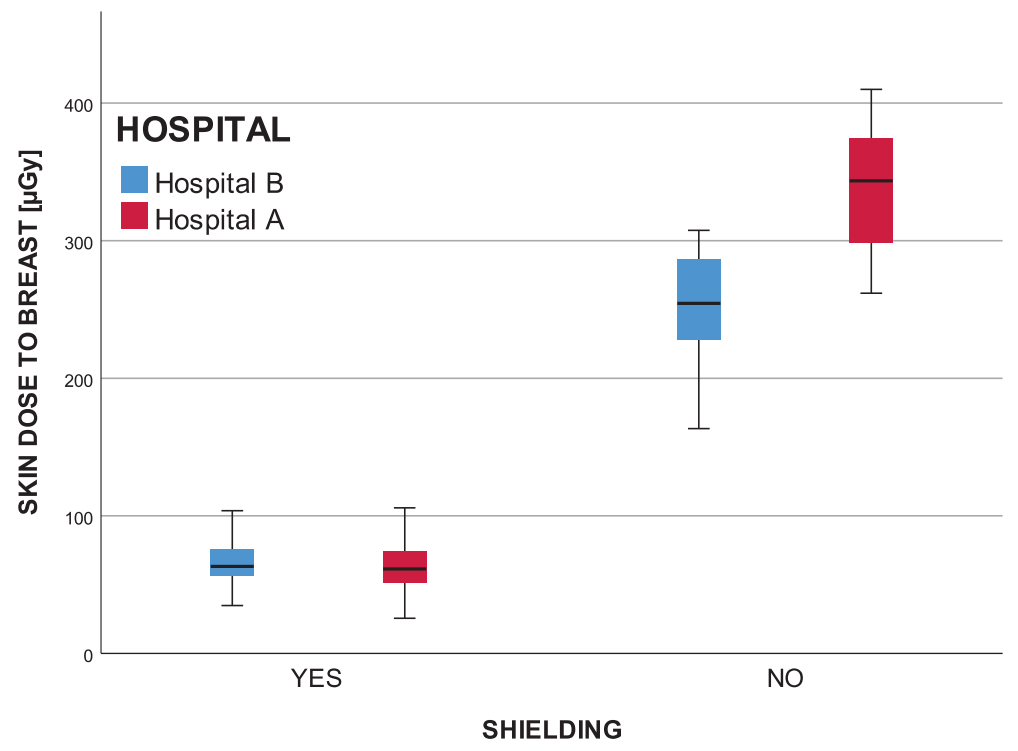

FIGURE 2. Mean skin dose to breast in both hospitals with and without the shielding.

Wrapped-around shielding is also the routine way of shielding in CT diagnostics' clinical practice, and not covering only one radiosensitive organ. Since shielded and unshielded measurements on the same patient could not be performed, a large sample was necessary for statistical accuracy. In total, 60 measurements of radiation dose to the breasts under the shielding were performed compared to the 20 measurements performed in the study by Beaconsfield et al. ${ }^{11}$ In the present study the measurements were performed in two different institutions on two different $\mathrm{CT}$ units with different exposure parameters. All the above contributes to the additional value of the present work.

Our study has proven that the use of lead shielding significantly reduced the skin dose to the breasts. Nevertheless, special attention still needs to be payed to the positioning of the apron. The lead shielding must be positioned so it does not enter the primary field. If the shielding enters the primary field of imaging, it would produce significant image artefacts or, if the automatic exposure control is used, the system would drastically increase the tube output in order to compensate the absorption in lead. Caution must also be taken to wrap the shielding around the patient as tightly as possible, so there is no air gap between the chest and the shielding.

This study was performed with a lead shield, which is the most commonly used form of shielding in Slovenia. New shielding materials are emerging, among them the Antimony-Bismuth shields, which 
are positioned in the primary field and only partially block the beam. Further investigation could be performed. Nevertheless, the breasts are located outside the primary field so the lead shielding can be applied to reduce the scattered radiation to the breasts.

This study showed that the lead shielding significantly reduced the amount of scatter radiation irradiating the breasts. The dose was reduced by a minimum of $74 \%$ and a maximum of $81 \%$. Head $\mathrm{CT}$ is one of the most frequently performed CT examinations and the breasts are one of the most radiosensitive organs so patients undergoing a head CT scan will benefit significantly when lead shielding is used. Our work could be continued by investigating the efficiency of the newly emerging shielding materials, its efficiency in correlation to material thickness and the frequency of using the shielding in different departments.

\section{References}

1. Akhlaghi P, Miri-Hakimabad H, Rafat-Motavalli L. Effects of shielding the radiosensitive superficial organs of ORNL pediatric phantoms on dose reduction in computed tomography. J Med Phys 2014; 39: 238-46. doi: $10.4103 / 0971-6203.144490$

2. International Atomic Energy Agency. Radiation in everyday life. [cited 2020 Mar 20]. Available at: https://www.iaea.org/Publications/Factsheets/ English/radlife.

3. Safiullah S, Patel R, Uribe B, Spradling K, Lall C, Zhang L, et al. Prevalence of protective shielding utilization for radiation dose reduction in adult patients undergoing body scanning using computed tomography. J Endourol 2017; 31: 985-91 doi: $10.1089 /$ end 2017.0294

4. European Commission. Radiaton protection $N^{\circ} 180$ medical radiation ex posure of the European population. Part 1 \& Part 2; 2015. [cited 2020 mar 20]. Available at: https://ec.europa.eu/energy/content/rp-180-medicalradiation-exposure-european-population-part-1-part-2 en

5. Williams L, Adams C. Computed tomography of the head: an experimental study to investigate the effectiveness of lead shielding during three scanning protocols. Radiography 2006; 12: 143-52. doi: 10.1016/j.radi.2005.05.001

6. Pace I, Zarb F. A comparison of sequential and spiral scanning techniques in brain CT. Radiol Technol 2015; 86: 373-8. PMID: 25835403

7. Valentin EJ, Boice Jr J, Meinhold C, Alexakhin R. Annals of the ICRP Publication 103: The 2007 Recommendations of the International Commission on Radiological Protection; 2007.

8. Brnić Z, Vekić $B$, Hebrang A, Anić P. Efficacy of breast shielding during $C T$ of the head. Eur Radiol 2003; 13: 2436-40. doi: 10.1007/s00330-003-1945-1

9. Sowby FD. Annals of the ICRP. Ann ICRP 1981; 6: 1. doi: 10.1016/01466453(81)90127-5

10. Preston DL, Mattsson A, Holmberg E, Shore R, Hildreth NG, Boice JD. Radiation effects on breast cancer risk: a pooled analysis of eight cohorts. Radiat Res 2002; 158: 220-35. doi: 10.1667/0033-7587(2002)158[0220:reo bcr]2.0.co;2

11. Beaconsfield T, Nicholson R, Thornton A. Would thyroid and breast shielding be beneficial in CT of the head? Eur Radiol 1998; 8: 664-7. doi: 10.1007/ s003300050456

12. Ngaile JE, Uiso CBS, Msaki P, Kazema R. Use of lead shields for radiation protection of superficial organs in patients undergoing head CT examinations. Radiat Prot Dosimetry 2008; 130: 490-8. doi: 10.1093/rpd/ncn095
13. Chung JJ, Cho ES, Kang SM, Yu JS, Kim DJ, Kim JH. Usefulness of a lead shielding device for reducing the radiation dose to tissues outside the primary beams during CT. Radiol Med 2014; 119: 951-7. doi: 10.1007/s11547-0140421-0

14. Zalokar N, Mekiš N. A phantom study showing the importance of breast shielding during head CT. Radiat Prot Dosimetry 2020; 186: 507-12. doi: $10.1093 / \mathrm{rpd} / \mathrm{ncz} 305$

15. Cardis E, Hall J, Tavtigian SV. Identification of women with an increased risk of developing radiation-induced breast cancer. Breast Cancer Res 2007; 9: 1-9. doi: $10.1186 /$ bcr1668

16. Mekis $\mathrm{N}$, Zontar $\mathrm{D}$, Skrk D. The effect of breast shielding during lumbar spine radiography. Radiol Oncol 2013; 47: 26-31. doi: 10.2478/raon-2013-0004 